\title{
A Model Based Continuous Improvement Methodology for Sustainable Manufacturing
}

\author{
Sanjay Jain ${ }^{1}$, Gordon $\mathrm{Shao}^{2}$, Alexander Brodsky ${ }^{3}$, and Frank Riddick ${ }^{2}$ \\ ${ }^{1}$ George Washington University, Washington, DC, USA \\ jain@email.gwu.edu \\ ${ }^{2}$ National Institute of Standards and Technology, Gaithersburg, MD, USA \\ \{guodong. shao, frank.riddick\} anist.gov \\ ${ }^{3}$ George Mason University, Fairfax, VA, USA \\ brodsky@gmu .edu
}

\begin{abstract}
This paper proposes a model based continuous improvement methodology to support efforts to achieve sustainable manufacturing, i.e., to increase efficiency and reduce environmental impact of manufacturing systems. Past efforts have provided guidance at a high level or with a focus on products. This paper focuses on supporting efforts for manufacturing, in particular, at the factory level. A framework is proposed to support the methodology by facilitating application of optimization and simulation models for sustainable manufacturing.
\end{abstract}

Keywords: Environment, energy, metrics, optimization, simulation, sustainable manufacturing.

\section{Introduction}

The global competition coupled with difficult world economic situation continues to push manufacturers to improve efficiency. There is also an increasing recognition of the need for sustainable manufacturing. Sustainable manufacturing includes efforts to reduce energy consumed, increase material efficiency (i.e., reduce material used per unit output), reduce water use, and reduce waste and emissions at manufacturing facilities.

Advancements in computing technology over recent years allow employing realistic optimization and simulation models to support manufacturing decision making. Technology for tracking of plant floor activities has further enabled the use of optimization and simulation models.

The confluence of the need for improving efficiency, reducing the use of earth's resources, and rapidly improving technology has motivated the proposal of a modelbased continuous improvement methodology and an associated framework for its use. The methodology is of a generic nature similar to the various continuous improvement methodologies including Deming's Plan-Do-Check-Act (PDCA) cycle. A domain specific infrastructure is required to implement the methodology.

Application of modeling and analysis techniques in general requires specialized skills that are usually not available in manufacturing environment. Successful 
implementation of the methodology hence requires an infrastructure that facilitates application of modeling and analysis for strategic and tactical manufacturing decisions. This paper proposes a framework for sustainability modeling and optimization that will provide the integrating infrastructure for developing models to improve the sustainability aspects of a manufacturing facility using the proposed methodology.

The next section reviews related prior efforts. Section 3 presents the methodology, while section 4 presents the proposed framework. The execution of the methodology using the framework is discussed in section 5. Section 6 concludes the paper.

\section{Related Efforts}

There have been multiple efforts for application of optimization and simulation models to improve sustainable manufacturing. Only a few relevant examples are mentioned here due to paper length constraints. Vergnano et al. (2012) utilized a mixed-integer linear programming model to optimize the schedule for a robotic manufacturing system leading to reduced energy consumption. Bi and Wang (2012) reduced the energy consumption of a selected machine tool through optimization of the process parameters. Taplin et al. (2006) utilized simulation to evaluate different options for improving the sustainability performance of a metal casting company. The sustainability metrics included amount of scrap, dross, and carbon dioxide emissions. Zhou et al (2011) utilized a simulation model together with a response surface methodology to identify the optimal solution with respect to green sustainable development measures including energy/resource conservation and environmental emission/pollution. The reported efforts employ significant expertise for developing and applying optimization and simulation models. There is a need for developing an infrastructure that facilitates such application.

Smith and Ball (2012) point out that most of the recent reported efforts in the area of sustainable manufacturing are at a high level, and thus there is limited guidance for improving sustainability at the factory level. They provide guidelines to analyze material, energy, and waste flows in a factory and use a quantitative spreadsheet model to identify improvements. The provided spreadsheet models facilitate Pareto analysis by assets and by flows to identify key areas for improvement. Improvement opportunities are evaluated using the quantitative and qualitative information gathered. Selected improvements are implemented using standard project management techniques. We are motivated by similar goals and propose a methodology and supporting infrastructure that allows application of advanced optimization and simulation models.

\section{Model Based Continuous Improvement Methodology}

The methodology is identified as "model based" as the analysis of current operations and improvements makes extensive use of various types of models including optimization and simulation. It is further identified as "continuous improvement" as it uses an iterative procedure employing an increasingly rich set of models to support 
successive improvements. This allows taking a leap beyond the typical process of adhoc models that end up as "shelf-ware" after a study. The proposed methodology can be executed for a manufacturing facility using the following steps as shown in figure 1.

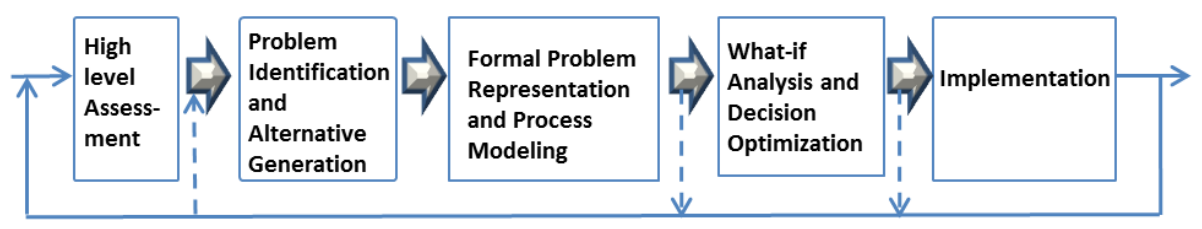

Fig. 1. Model Based Continuous Improvement Methodology

High Level Assessment: This step is aimed at upfront identifying the area where the improved planning effort should be focused on. This step will focus on a high level assessment of the major opportunities for improvements using identified metrics. In manufacturing applications, this step may be referred to as "factory level assessment." For example, based on comparison with other facilities of similar nature, this step may identify that the largest improvement opportunity for improving sustainability of a facility is via reducing its energy consumption. This assumes that the facility has a system in place for collecting a number of different metrics and for comparing them with other facilities.

Problem Identification and Alternative Generation: This step includes the collection of data and analysis for narrowing down the area targeted for improvement effort and development of an improvement plan. The collected data relevant to the narrowed down area will help identify the problem specifically. Alternatives may be generated based on experience and knowledge of the team. The team may suggest policy, procedure, process, and/or equipment modifications to achieve desired improvements. If the factory level assessment identified reducing energy consumption as the primary focus, this step may narrow that down to reducing energy consumption in heating and cooling of the facility based on comparison with other uses of energy and potential reductions. Subsequently alternatives for the purpose may be generated including, adding insulation to the building, adding thermal enclosures around manufacturing equipment that generate large amounts of heat, and improving efficiency of HVAC systems.

Formal Data Representation and Process Modeling: This step involves representation of the manufacturing process and facility data using proposed structures that allow capturing the current state to develop a better understanding of the underlying phenomenon and causal relationships. Once the requisite data is represented using proposed structures, it can be used to generate optimization and/or simulation models of the areas of interest of the manufacturing system. The simulation modeling part of this step can be viewed as equivalent to the "AS-IS" modeling of the process reengineering efforts. Continuing the energy consumption reduction example, a simulation model of the manufacturing facility with representative product volumes processed through equipment of interest will allow verification of the ranges of 
amounts of generated heat. The heat generated from manufacturing equipment can then be used in the heat flow calculations to estimate the heating, ventilation and cooling (HVAC) energy demand and compared against recorded values. This ensures that the AS-IS model has captured the relevant major factors and serves to validate the simulation model for the next step. The insights gained at this step may uncover a problem that needs to be addressed first leading to a loop back to problem identification step as shown in figure 1.

What-If Analysis and Decision Optimization: This step will involve development of optimization and simulation models for generation and evaluation of the improvement alternatives respectively. The evaluation will include use of metrics generated by the models and business metrics such as return on investment. Optimization models may be used to develop proposed solutions to achieve the desired goals. Alternatives may also be generated by the decision makers and analysts based on the past experience and the increased understanding and insights gained through the development and use of simulation models of the current processes. For example, optimization may be used to determine the combination of available options that will best achieve the sought for energy reduction. It may identify the proportion of products to be produced using alternative processes such that the generated heat is minimized.

Simulation models can be used to evaluate "what-if" alternatives generated by decision makers and analysts. They can also be used to validate the alternatives generated by the optimization models since more often than not the optimization models cannot take all the realistic factors into account.

The analysis at this step may determine that none of the proposed alternatives provide a desired return and result in a loopback to the problem identification step.

Implementation: This step involves implementing the improvement plan validated via simulation in the previous step. For the discussed example, it may involve changing parameters in the production scheduling system such that desired proportion of products flows through the alternative processes.

The methodology is generic and can be applied to different domains though it has been explained above with respect to the manufacturing environment. Implementation of the methodology is challenging and requires high expertise with large time and effort particularly for the modeling and analysis steps. The challenges can be significantly reduced through the use of an infrastructure that facilitates development of models appropriate to the domain. The framework described in the next section is aimed at providing just such an infrastructure.

\section{$4 \quad$ Framework for Sustainability Modeling and Optimization}

The proposed framework for sustainability modeling and optimization (SMO) provides an integrating infrastructure for capabilities needed for development of models of manufacturing that include sustainability aspects. It is comprised of four major components described below. The framework will support a test-bed that will utilize 
commercial-off-the-shelf (COTS) simulation and optimization tools to support sustainability analysis of factories (see Lee et al., 2013 for the test-bed concept).

\subsection{Sustainable Manufacturing Maturity Model (SM3)}

The sustainable manufacturing maturity model (SM3) will be used to support the "high level assessment" step of the methodology, i.e., to conduct the factory level assessment to gauge the current sustainability performance possibly against a set of defined levels and determine the improvement opportunities using the next level of maturity as the target. An initial version of the model is show in figure 2. The first and second levels assess the ability of organization to capture standard metrics for energy use, material efficiency, and corporate carbon footprint. Levels III and IV assess and guide the factory's journey to improved sustainability. Clearly, this approach has to address the major challenge of identifying the best in class. Further work is in progress to develop alternate approaches.

\begin{tabular}{|c|l|}
\hline Level & \multicolumn{1}{|c|}{ Criteria } \\
\hline IV & Metrics among best in class \\
\hline III & Metrics halfway to the best in class from base values \\
\hline II & Metrics base values captured using standard methods \\
\hline I & Environmental Management System implemented \& audited \\
\hline $\mathbf{0}$ & Status quo \\
\hline
\end{tabular}

Fig. 2. An initial version of Sustainability Manufacturing Maturity Model

\subsection{Sustainable Process Description Model (SPDM)}

A unified sustainable process description model will be developed to support the "Model" step of the methodology, i.e., for creating a formal representation that captures the factory information and sustainability objectives needed to develop models of alternative solutions to the problem (Shao et al., 2012). Figure 3 presents the current concept of the description model.

Initially diverse manufacturing processes and industrial scenarios will be examined to identify most appropriate modeling methods/tools/ languages, and to specify the process representation requirements. A representation will be designed to address the research challenges of (1) providing a generic mechanism to represent the diverse manufacturing scenarios, and (2) supporting taxonomies and metrics for representation of unit manufacturing and assembly processes. Specifically, the process representation will be designed to include process structure and data model to describe input and output resources, sustainability data, and hierarchical composition of processes. 


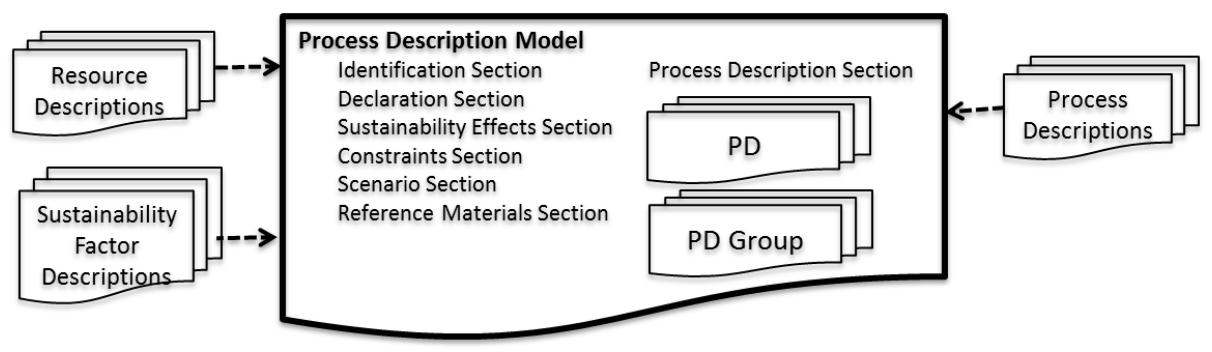

Fig. 3. Concept of Sustainability Process Description Model

\subsection{Sustainable Process Analytical Formalism (SPAF)}

A process analytics formalism will be used to support the "Check" step of the methodology, i.e., for "what-if" analysis and decision optimization. The formalism and associated procedures will automatically translate the analytical representation into a formal optimization model that can be executed using commercial optimization software. The formalism will be designed to include process analytics model to define control variables, express metrics computation, and define constraints that completely characterize a set of process alternatives. A prototype system for translating the analytical representation to a formal optimization model has been implemented (Shao et al. 2013).

The range of problems faced by industry in improving sustainability will be studied to define model scope and answer questions. The research challenge here is the development of sound and complete methods to automatically transform decision optimization questions posed against the sustainable process analytical representation into formal optimization models. These machine-generated models will then be solved by COTS optimization tools selected for the proposed test-bed.

\subsection{Support Tools}

Support tools and procedures may be used to facilitate various steps. In particular, they include translators that may be used to generate optimization/simulation models that can be executed using COTS software.

Figure 4 shows the integration of components of framework to facilitate sustainability modeling and optimization by human decision makers and analysts. The maturity model will help identify the improvement opportunities areas that can be delved into to identify specific objectives for an improvement initiative and associated constraints. The understanding of the problem can help develop a conceptual model of the factors involved that in turn guides the data to be collected to further analyze the problem. The conceptual model of the problem may be formally represented using the description model. Support tools may use the description model representation to generate simulation models that can be executed using commercial simulation software. The simulation models can be used to study the problem. 
Once the data is represented in the description model, support tools can be used to facilitate representation of optimization problem using the formalism. Again, support tools can be used to execute the optimization using commercial optimization and analysis tools.

The results from the simulation and optimization models can be presented to the human decision makers and analysts to help guide the decision making.

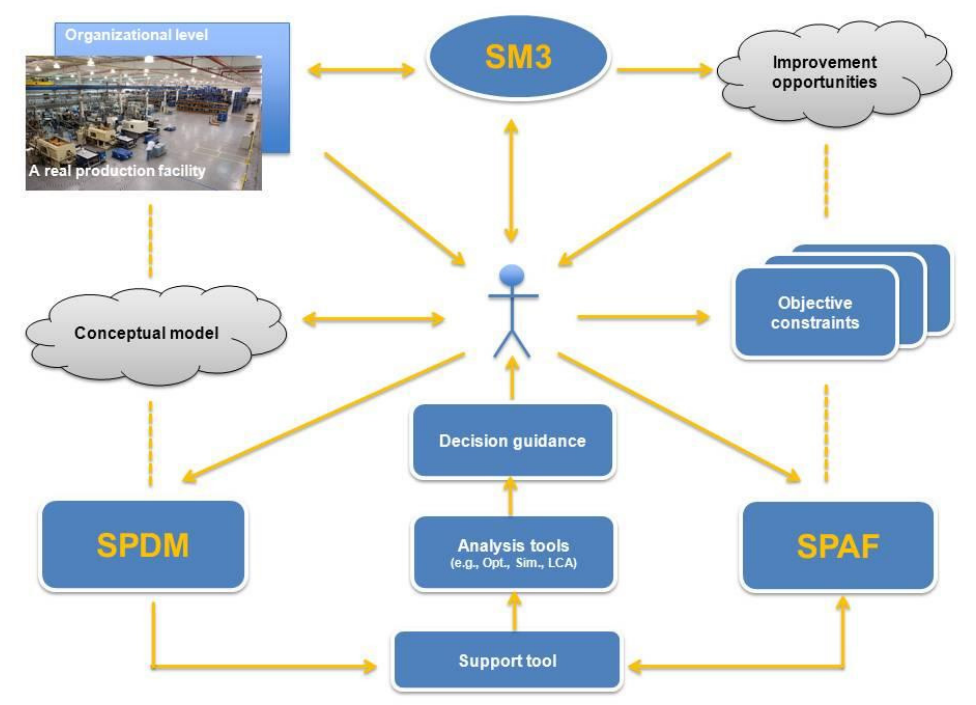

Fig. 4. Framework for Sustainability Modeling and Optimization for Decision Guidance

\section{$5 \quad$ Methodology Execution Using SMO Framework}

The execution of the methodology can be facilitated, indeed enabled, through the use of the framework as shown in figure 5. The first step, high level assessment, will be supported by the maturity model component of the framework. The maturity model will help a manufacturing facility identify the metric that offers the opportunity for most improvement. The metrics may include energy use, material efficiency, and carbon footprint. The next step of problem identification and alternative generation will then focus on the metrics identified via the high level assessment. This step will require collection of data to identify the relevant operations within the factory that have a large impact on the selected metric.

To prepare for formal analysis and optimization modeling, defined case scenarios need to be formally described, and data collected for the defined case scenarios need to be formally represented. Further, decision variables need to be modeled in a way so that their values can be automatically instantiated, i.e., assigned using the appropriate data items. The formal representation and process modeling step will be supported by the description model and the formalism components of the framework. The collected data is represented using the description model. The structures in the 
description model may identify other relevant data needed for process modeling and thus prompt correct and complete data collection. The formalism supports these by representing process analytics expression such as mathematical specification for metrics, constraints, and objectives; and enabling the formulation of what-if analysis and decision optimization queries.

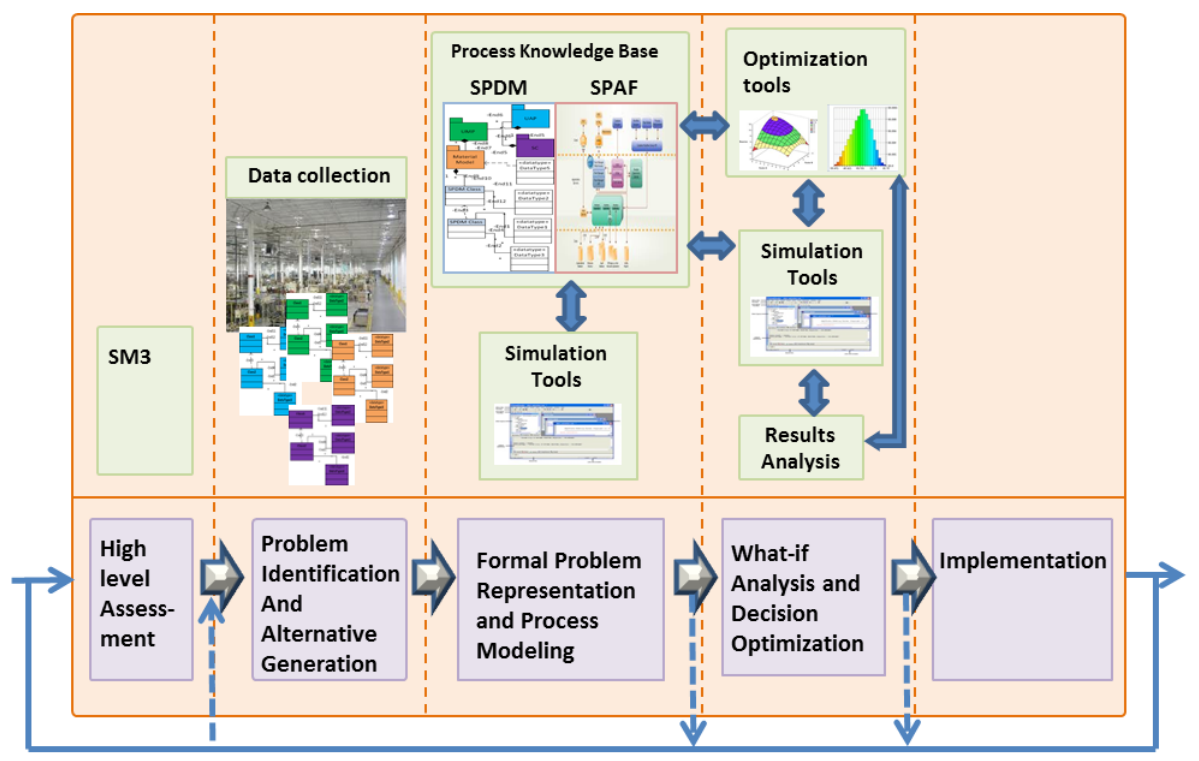

Fig. 5. Methodology execution using SMO framework

The description model representation also provides sustainability indicators and metrics for the formalism representation of the problem through the framework support tools. The support tools in the framework are then used to generate simulation models of the current state of the factory in COTS simulation software. The simulation models are used to develop further understanding of issues surrounding the problem. In some cases sufficient understanding may exist to move to the next step quickly while in others a number of simulation runs may be executed to understand the key factors that impact the metrics of interest. Queries using the formalism also facilitate the understanding of the problem at this stage. Users may query the system for questions ranging from simple ones such as the process for a particular product to more complex ones such as the percentage contribution to energy use by a particular product flow at particular equipment.

The what-if analysis and decision optimization step of the methodology is supported by the framework using the description model and the formalism representations, support tools, and COTS simulation and optimization tools. Two kinds of queries using the formalism are used at this step: what-if analysis queries and decision optimization queries. Different answers to what-if analysis allow decision makers to compare and assess changes before they happen; users can see how different changes 
would affect various aspects of the outcome. For example, given certain settings of a process' controls and input, total carbon emission and costs can be estimated before investing in a new machine tool. If all variables in the expressions can be instantiated through computation using the data given, What-if analysis queries using the formalism allow computation and simulation representing different alternatives and analysis of outputs to determine the alternatives that help achieve the desired performance improvement.

Decision optimization queries using the formalism can be translated to a standard optimization model such as an Optimization Programming Language (OPL) model, which can be solved using an optimization solver such as IBM ILOG CPLEX. If the problem is feasible and valid, the optimal solution will provide actionable recommendations to decision makers. For example, given certain production requirements, determine process control parameters that minimize carbon emissions within a given cost, or minimize cost within given emission bounds. Optimization can combine with simulation in case where the optimization problem representation does not take into account all the detailed factors of real life operations.

The implementation step can proceed as before except with the benefit of results of models the team would have a higher level of confidence and comfort. Similar to the recommendation by Smith and Ball (2012), the implementation effort will gain from use of standard project management techniques. Once the implementation is complete, the next iteration of improvement can be initiated with the high level analysis.

\section{Conclusion}

This paper described a model based continuous improvement (MBCI) methodology that can be utilized by manufacturers for supporting their movement towards improved sustainability. The model based nature of the methodology allows manufacturers to evaluate alternative approaches in virtual mode, i.e., using computer models, and this provides a more efficient approach than the traditional plan-do-check-act improvement cycle. The efficiency comes from avoiding the time and expense of a real life pilot of a selected alternative for its evaluation and instead allowing evaluation of multiple alternatives via rapid modeling. The paper also proposed a framework for Sustainability Modeling and Optimization to provide an integrated set of capabilities for modeling and optimization of manufacturing systems. The paper further discussed how the implementation of the MBCI methodology can be enabled by the framework.

\section{Disclaimer}

A number of software products are identified in context in this paper. This does not imply a recommendation or endorsement of the software products by the authors or NIST, nor does it imply that such software products are necessarily the best available for the purpose. 


\section{References}

1. Bi, Z.M., Wang, L.: Energy Modeling of Machine Tools for Optimization of Machine Setups. IEEE Transactions on Automation Science and Engineering 9(3), 607-613 (2012)

2. Lee, Y.-T.T., Lee, J.Y., Riddick, F., Libes, D., Kibira, D.: Interoperability for Virtual Manufacturing Systems. International Journal of Internet Manufacturing and Services (forthcoming, 2013)

3. Shao, G., Riddick, F., Lee, J.Y., Campanelli, M., Kim, D.B., Lee, Y.T.: A Framework for the Interoperability of Sustainable Manufacturing Process Analysis Applications. In: 2012 Winter Simulation Conference. IEEE, Piscataway, http://informs-sim.org/wsc12papers/includes/files/con334.pdf

4. Shao, G., Westbrook, D., Brodsky, A.: A Prototype Web-Based User Interface for Sustainability Modeling and Optimization. NIST Interagency/Internal Report (NISTIR) - 7850 (2013), http: / /www.nist.gov/customcf/get_pdf.cfm?pub_id=909618

5. Smith, L., Ball, P.: Steps towards sustainable manufacturing through modelling material, energy and waste flows. Intl. Journal of Production Economics 140(1), 227-238 (2012)

6. Taplin, D.M.R., Spedding, T.A., Khoo, H.H.: Use of simulation and modelling to develop a sustainable production system. Sustainable Development 14(3), 149-161 (2006)

7. Vergnano, A., Thorstensson, C., Lennartson, B., Falkman, P., Pellicciari, M., Leali, F., Biller, S.R.: Modeling and Optimization of Energy Consumption in Cooperative Multi-Robot Systems. IEEE Trans. on Automation Science and Engg. 9(2), 423-428 (2012)

8. Zhou, M., Pan, Y., Chen, Z., Yang, W., Li, B.: Simulation based analysis for selection and evaluation of green manufacturing strategies. In: 8th International Conference on Service Systems and Service Management (ICSSSM 2011), pp. 1-6 (2011) 